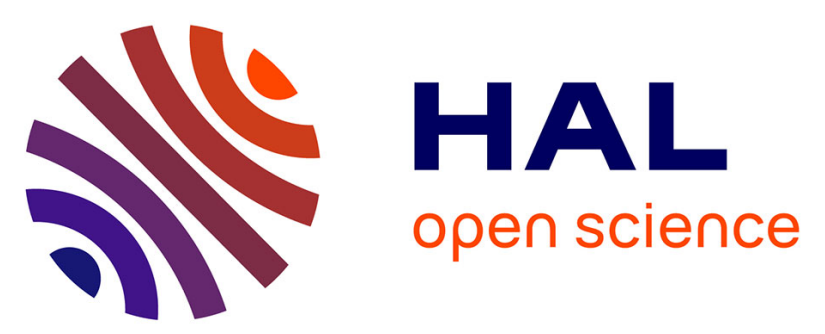

\title{
Chemisorption of Hydroxide on 2D Materials from DFT Calculations: Graphene versus Hexagonal Boron Nitride
} Benoit Grosjean, Clarisse Pean, Alessandro Siria, Lydéric Bocquet, Rodolphe Vuilleumier, Marie-Laure Bocquet

\section{To cite this version:}

Benoit Grosjean, Clarisse Pean, Alessandro Siria, Lydéric Bocquet, Rodolphe Vuilleumier, et al.. Chemisorption of Hydroxide on 2D Materials from DFT Calculations: Graphene versus Hexagonal Boron Nitride. Journal of Physical Chemistry Letters, 2016, 7 (22), pp.4695 - 4700. 10.1021/acs.jpclett.6b02248 . hal-01419901

\section{HAL Id: hal-01419901 https: / hal.sorbonne-universite.fr/hal-01419901}

Submitted on 20 Dec 2016

HAL is a multi-disciplinary open access archive for the deposit and dissemination of scientific research documents, whether they are published or not. The documents may come from teaching and research institutions in France or abroad, or from public or private research centers.
L'archive ouverte pluridisciplinaire HAL, est destinée au dépôt et à la diffusion de documents scientifiques de niveau recherche, publiés ou non, émanant des établissements d'enseignement et de recherche français ou étrangers, des laboratoires publics ou privés. 


\section{Chemisorption of Hydroxide on 2D Materials}

\section{From DFT Calculations: Graphene Versus}

\section{Hexagonal Boron Nitride}

Benoit Grosjean, ${ }^{\dagger}$ Clarisse Pean,${ }^{\dagger}$ Alessandro Siria, ${ }^{\ddagger}$ Lyderic Bocquet, ${ }^{\ddagger}$ Rodolphe Vuilleumier, ${ }^{\dagger}$ and Marie-Laure Bocquet ${ }^{\dagger}$

†École Normale Supérieure-PSL Research University, Département de Chimie, Sorbonne Universités - UPMC Univ Paris 06, CNRS UMR 8640 PASTEUR, 24, rue Lhomond, 75005 Paris, France

$\ddagger E$ École Normale Supérieure-PSL Research University, Laboratoire de Physique Statistique, UMR 8550, 24, rue Lhomond, 75005 Paris, France 


\begin{abstract}
Recent nanofluidic measurements revealed strongly different surface charge measurements for boron-nitride and graphitic nanotubes when in contact with saline and alkaline water. ${ }^{12}$ These observations contrast with the similar reactivity of a graphene layer and its boron nitride counterpart, using Density Functional Theory (DFT) framework, for intact and dissociative adsorption of gaseous water molecules. Here, we investigate, by DFT in implicit water, single and multiple adsorption of anionic hydroxide on single layers. A differential adsorption strength is found in vacuum for the first ionic adsorption on the two materials - chemisorbed on BN while physisorbed on graphene. The effect of implicit solvation reduces all adsorption values resulting in a favorable (non-favorable) adsorption on $\mathrm{BN}$ (graphene). We also calculate a $p K_{a} \simeq 6$ for $\mathrm{BN}$ in water, in good agreement with experiments. Comparatively, the unfavorable results for graphene in water echoes the weaker surface charge measurements, but points to an alternative scenario.
\end{abstract}

\title{
Graphical TOC Entry
}

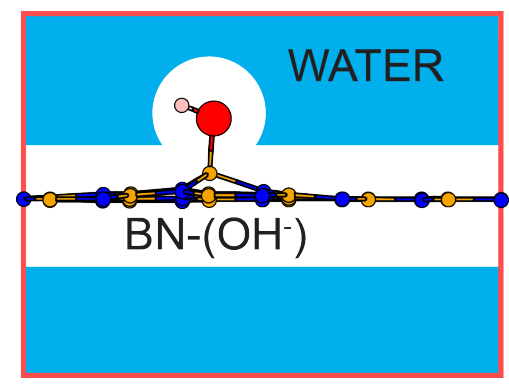


The successful isolation of single-layer graphene sheet ${ }^{[3}$ has led to tremendous progress in the discovery of new 2D materials including boron nitride, silicene and transition metal dichalcogenides to cite a few. In particular hexagonal boron nitride monolayers share the planar honeycomb structure of graphene, with boron and nitrogen atoms alternating in the vortices of the honeycomb structure. Despite of their similar crystallographic structure, the electronic structure of $\mathrm{BN}$ and graphene nano sheets are drastically different : semimetallic for graphene and insulating for BN. Until recently both pristine layered materials were assumed to present inherently low chemical reactivity. However recent experimental works showed that covalent chemical fonctionalization could be achieved under drastic reactive conditions, either via reduction ${ }^{4 \sqrt{45}}$ or reaction with oxidative reagents $\left(\mathrm{H}_{2} \mathrm{O}_{2}, \underline{6}\right.$ oxygen radical ${ }^{7}$ ) or via fluorination. ${ }^{[8}$ Furthermore recent nanofluidic experiments have altered the long-standing picture of the chemical inertness of BN nanotubes by showing a large negative surface charge when the BN materials is in contact with aqueous saline solutions. In contrast, similar experimental investigations based on nanofluidic investigations showed that carbon nanotubes ${ }^{2}$ and planar graphitic surfaces ${ }^{9}$ exhibit smaller and even minute surface charge.

The magnitude of the maximum charging - ranging from $0.1 \mathrm{C} / \mathrm{m}^{2}$ for $\mathrm{C}$ to $1 \mathrm{C} / \mathrm{m}^{2}$ for $\mathrm{BN}$ depending on the solution $\mathrm{pH}$ - rules out point defects as the sole origin of the measured surface charging. These experimental results therefore call for two challenging questions: (i) what are the potential chemical species present in basic and saline solutions that could adhere on the surface and strongly charge the pristine nanotubes? (ii) how can we explain the different charging behavior of graphene and BN ideal nanotubes?

A recent DFT study has investigated the dissociation barrier of a water molecule in contact with pristine graphene and BN nano sheets and concluded that the process is highly endothermic with a larger barrier on graphene than on BN.10 They also proposed facile ways to enhance the dissociation, either via including substitutional defects or either via pre-adsorption of $\mathrm{H}$, which again amounts to the presence of defects. 
Here in this DFT work we consider pristine nano sheets and take into account the presence of hydroxide ions in water. We demonstrate that adding one electron (via the charged adsorbate) to these $2 \mathrm{D}$ materials permits to tune the adsorption strength of one hydroxide : a strong ionic adsorption on $\mathrm{BN}$ and a weak ionic adsorption on graphene. Our atomistic results corroborate the acidity $\left(\mathrm{pK}_{a}\right)$ of the $\mathrm{BN}$ sheet, pointing to the hydroxide anion as the best candidate to explain the charging of $\mathrm{BN}$ material immersed in a saline electrolyte.

A)

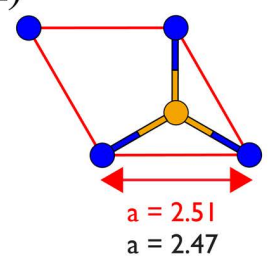

C)

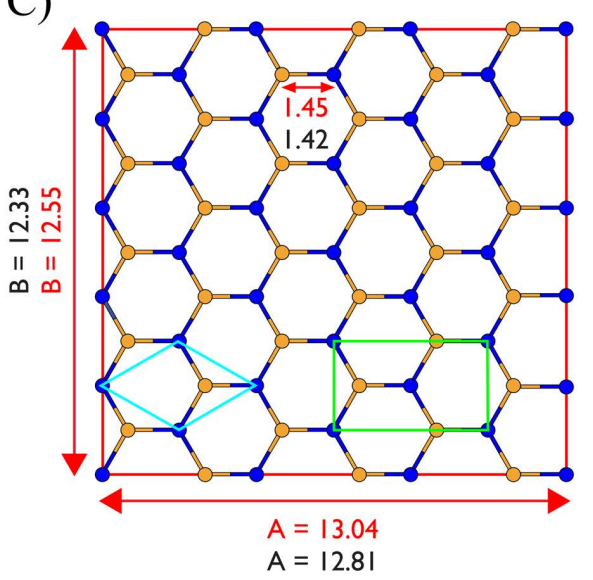

B)

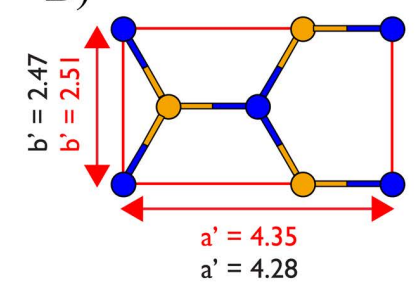

D)

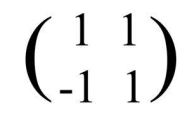

E)

$\left(\begin{array}{rr}3 & 5 \\ -3 & 5\end{array}\right)$

Figure 1: (A) BN hexagonal primitive unit cell. (B) BN Orthorhombic primitive unit cell. (C) BN orthorhombic supercell adopted in the DFT calculations. Distances, expressed in $\AA$, are written in red (black) for the case of BN (graphene). Boron, Carbon, Nitrogen and Oxygen are respectively displayed in yellow, grey, blue and red.(D) is the matrix to transform cell (A) into cell (B) and (E) the matrix to obtain cell (C) from cell (A).

The total energies of the systems have been calculated using the periodic DFT code Vienna Ab initio Simulation Package VASP (see Methods). The graphene and h-BN single layers were modeled using the same supercell corresponding to an $(3 \times 5)$ orthorhombic unit cell containing 60 atoms in Periodic Boundary Conditions (PBC) (see figure 1). The slab monolayer is separated by a vacuum space of $15 \AA$ to avoid the interaction between the 

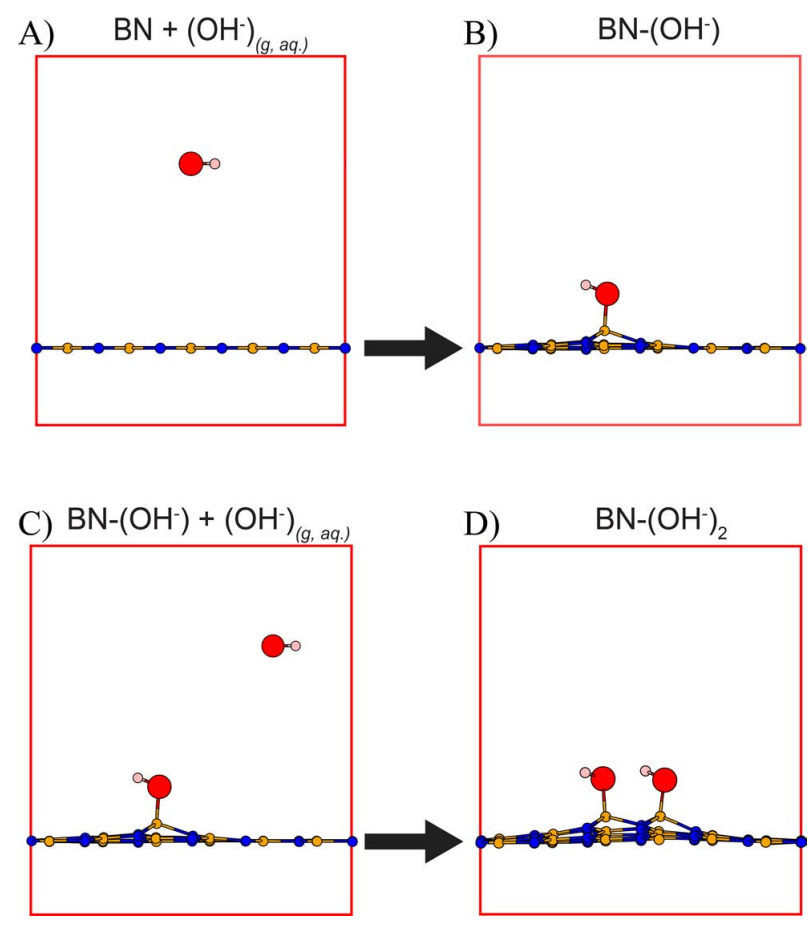

Figure 2: (top) Simulation cells for the BN monolayer (same on graphene not shown) used to determine the energy of the adsorption of the first anionic hydroxide. (A) Sub $+\mathrm{OH}_{f}$ : substrate and one desorbed anionic hydroxide in gaseous or aqueous phase. (B) $\mathrm{OH}_{a}$ : substrate with an adsorbed hydroxide onto either a $\mathrm{B}$ atom (BN layer) or a $\mathrm{C}$ atom (graphene layer). (bottom) Simulation scheme used to determine the energy of the adsorption of a second hydroxide $(\mathrm{C}) \mathrm{OH}_{a}+\mathrm{OH}_{f}$ : a surface with an adsorbed hydroxide and a desorbed hydroxide in gaseous or aqueous phase. (D) $2 \mathrm{OH}_{a}$ : a substrate with two adsorbed hydroxides. 
periodic images. The gamma point has been used in the k-point sampling of the first Brillouin zone of the supercell. The graphene and h-BN bond distances are evaluated to $1.43 \AA$ and $1.45 \AA$ with the PBE functional in agreement with previous calculations with the same method.11 We study the adsorption of anionic hydroxide molecule onto one B (or C) atom of the $\mathrm{sp}^{2} \mathrm{BN}$ (graphene) layer respectively. The resulting adsorption state is therefore negatively charged and an homogeneous background charge is automatically added in the periodic supercell to ensure its neutrality. As a result the total energy of a charged solid system includes an additional electrostatic interaction with the charged background. To compute the first adsorption energy of $\mathrm{OH}^{-}$called $E_{a d s 1}$, we consider the adsorbed $\mathrm{OH}$ on a negatively charged surface (by adding an extra electron to the total valence electrons) as the final state and the non-interacting species taken in the same supercell as the initial state as shown in Figure 2A-B. By doing so, the additional electrostatic interaction with the charged vacuum cancels out in the resulting adsorption energy.

$$
E_{a d s 1}=E\left(O H_{a}\right)-E\left(O H_{f}\right),
$$

where the indices $a$ and $f$ stand for adsorbed and desorbed $\mathrm{OH}^{-}$respectively (see figure caption 21). The second adsorption energy proceeds similarly as illustrated in Figure 2 C-D.

$$
E_{a d s 2}=E\left(2 O H_{a}\right)-E\left(O H_{a}+O H_{f}\right)
$$

Table 1 summarizes the energetics computed within periodic DFT for the first $\mathrm{OH}^{-}$adsorption on pristine BN and graphene monolayers subjected to different environments. In vacuum the adsorption is strongly favorable with $-2.3 \mathrm{eV}$ on $\mathrm{BN}$ layer, while it is less favored on the graphene layer $(-0.5 \mathrm{eV})$. The obtained values are strikingly different for both materials. The difference can be assigned to the charged ligand. Comparatively the neutral adsorption computed with spin-polarized calculations leads to similar moderate values (around $-0.6 \mathrm{eV})$ in full agreement with previous studies. ${ }^{10112}$ Interestingly the adsorption values do 
not depend on the inclusion, or not, of van der waals (vdw) corrections ( $\mathrm{see} \mathrm{E}_{a d s 1}(\mathrm{vdw})$ in Table 1). Indeed in the final adsorption state the species are short-range bonded precluding vdw interactions and in the initial desorbed state the species are positioned to avoid any spurious vdw interaction.

In contrast the adsorption values in implicit water are greatly reduced by around $1 \mathrm{eV}$ maintaining a favorable adsorption value for $\mathrm{BN}$ while turning to an unfavorable adsorption value for graphene. Notably including the solvent in the DFT modelling permits to discriminate both materials regarding the adsorption of one anionic hydroxyl : BN will spontaneously attach, while graphene should remain inert at least at low temperatures. We note in passing an alternative way to model the adsorption of a charged ligand with zero net charge in the supercell : the insertion of a counterion such as one potassium cation in the vacuum space at long distance from the adsorbate and the surface. The resulting energetics including the potassium cation are very similar to the charged procedure used in this study (see Supporting Information). Hence the discriminative energetics between the two materials are robust against different modeling schemes of the charging effect.

Table 1: Adsorption energies (in eV) of the first $\mathrm{OH}^{-}$species onto $\mathrm{BN}$ and Graphene layers in vacuum and in implicit water. Negative (positive) values mean favorable (unfavorable) cases. For the adsorption in vacuum, values in parentheses are spin-polarized calculation for the uncharged system, i.e the radical $O H$ adsorption that induces magnetism in graphene with $\mathbf{M}$ the electron magnetism.

\begin{tabular}{lcc}
\hline \hline$O H^{-}(O H \cdot)$ & BN layer & Graphene layer \\
\hline$E_{a d s 1}$ & $-2.3(-0.63, \mathrm{M}=1)$ & $-0.5(-0.57, \mathrm{M}=1)$ \\
$E_{a d s 1}^{\text {sol }}$ & -0.89 & +.33 \\
\hline$E_{a d s 1}(v d w)$ & -2.1 & -0.52 \\
$E_{a d s 1}^{\text {sol }}(v d w)$ & -0.9 & +.33 \\
\hline
\end{tabular}

The detailed DFT geometries of the adsorbed structures are displayed in Figure 3. Side views permit to visualize the $\mathrm{sp}^{3}$ rehybridization of the bonding atom inducing an out-ofplane buckling somewhat larger for BN than for graphene. The bonding distances between 
the $\mathrm{O}$ atom and the anchoring site on the substrates are $1.5 \AA$ for $\mathrm{BN}$ and $1.54 \AA$ for graphene, typical for single C-O bonds. A pyramidalization angle $\theta_{p}^{13}$ can be determined and amounts to $18^{\circ}$ for $\mathrm{BN}$ and $15^{\circ}$ for graphene corresponding to $93 \%$ and $77 \%$ of $\mathrm{sp}^{3}$ hybridization respectively. The geometrical differences are not as striking as the energy differences but it confirms a weaker adsorption of $\mathrm{OH}^{-}$on graphene than on $\mathrm{BN}$.
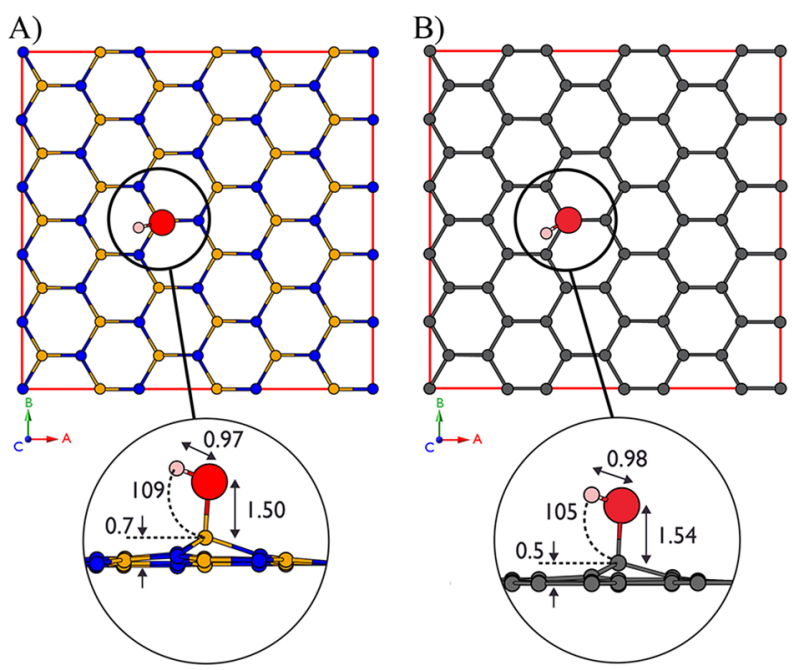

Figure 3: DFT adsorption structures. Top views of BN (A) and graphene (B). Insets with side views of the bonding site for each material with key distances and angles expressed in $\AA$ and degrees, respectively.

We have investigated the spatial distribution of the additional electron using the Mulliken charge analysis (see Methods). It strongly differs between BN and graphene nano sheets (see supplementary Table). The grafted $\mathrm{C}$ atom on graphene gains only 0.06 electron and the rest of $\mathrm{C}$ atoms remains practically non-charged. On graphene the additional charge is totally delocalized over the nano sheet and the possible charge-activating role to bind hydroxyl is therefore masked. In contrast, on $\mathrm{BN}$ the grafted $\mathrm{B}$ gains 0.22 electron and becomes less positively charged than the rest of electron-deficient B atoms. The additional charge on BN is therefore substantially localized on the ligand and the grafted atom and the local reduction of the binding $\mathrm{B}$ atom can explain its improved reactivity.

We then studied the adsorption of a second $\mathrm{OH}^{-}$ion onto $\mathrm{BN}$ and graphene monolayers. Figure 4 displays the second adsorption energy in vacuum $\mathrm{E}_{a d s 2}$ (dashed lines) and in implicit 
A)

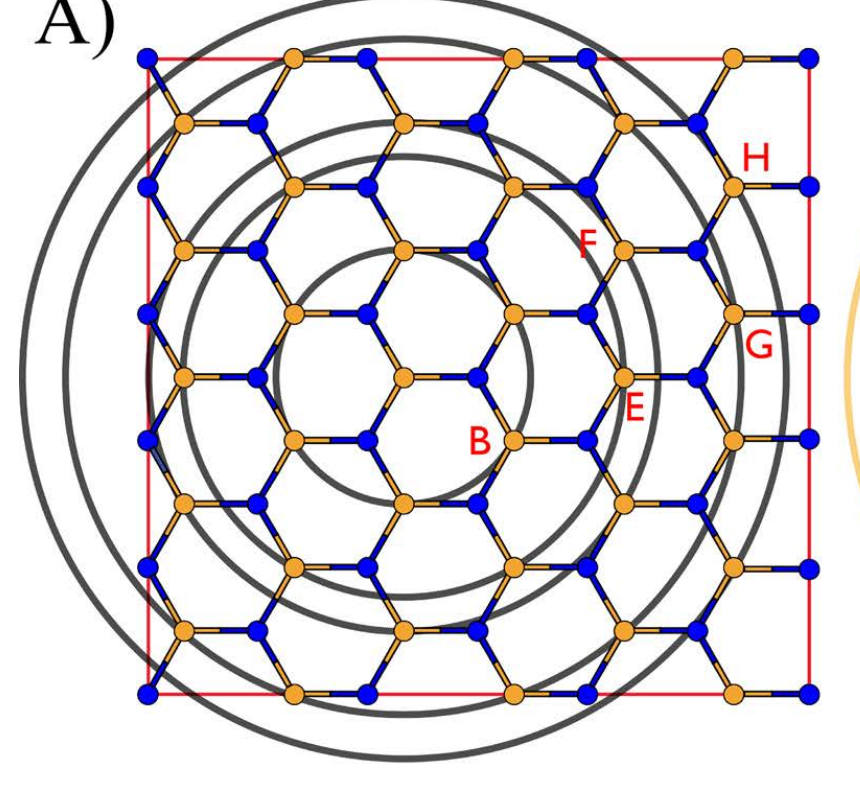

B)

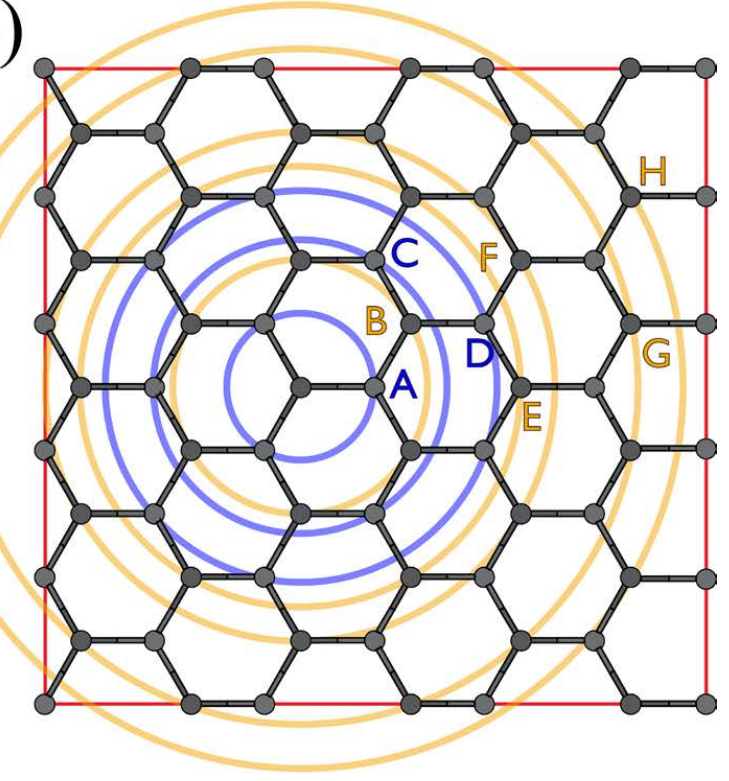

C)

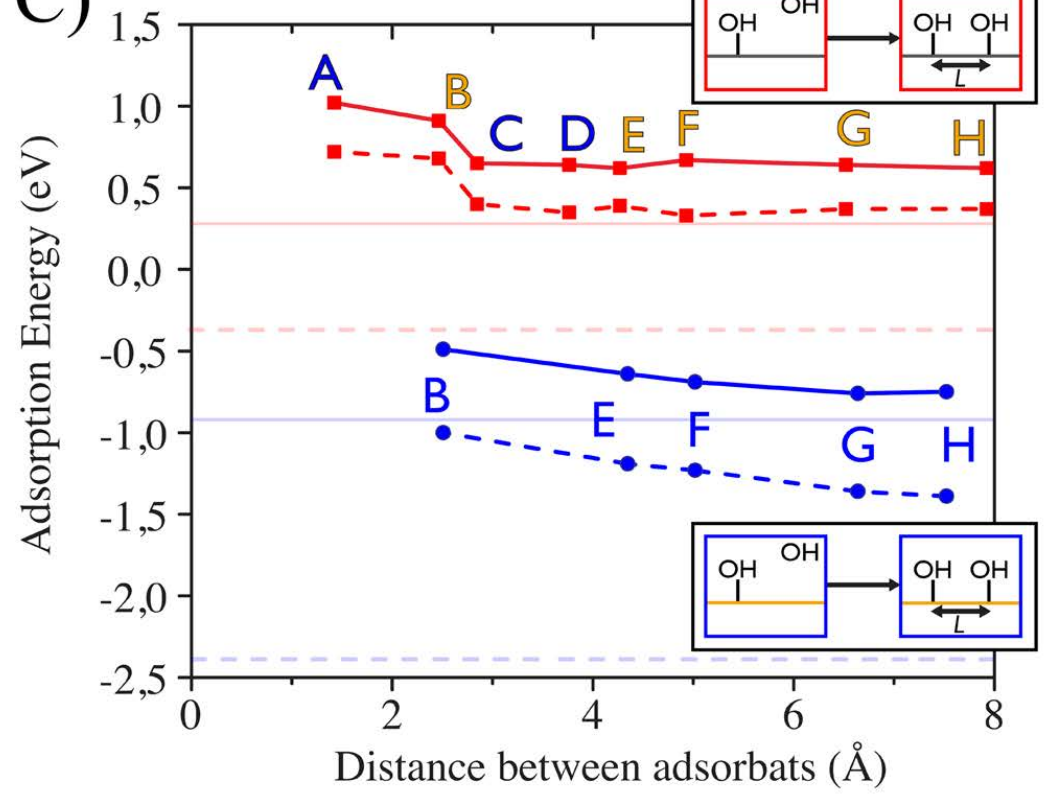

Figure 4: A) Circles display possible sites (labelled with letters) for the second adsorption of $\mathrm{OH}^{-}$at a given distance from the first adsorption site labelled A. B) Same for graphene. C) Adsorption energy of a second hydroxide versus the distance between the two adsorption sites. Results for BN are displayed by circles connected by blue lines while results for graphene are represented by squares connected by red lines. Horizontal lines represent the adsorption energies for single $\mathrm{OH}^{-}$ions (blue for $\mathrm{BN}$, red for graphene). Plain and dashed lines respectively correspond to adsorptions in implicit water and in vacuum. 
solvant $\mathrm{E}_{\text {ads } 2}^{\text {sol }}$ (solid lines) as a function of the distance $L$ from the first adsorption site. Circles mark the successive next nearest neighbors. Because only every two atoms are available for $\mathrm{OH}^{-}$adsorption on h-BN, there are twice more of such sites on graphene as compared to BN.

As shown on Fig. 4, the addition of a second $\mathrm{OH}^{-}$ion on the nearest neighbor located in the same hexagon destabilizes the adsorption energy by typically $\sim 1 \mathrm{eV}$ as compared to the single ion adsorption. It remains favorable for $\mathrm{BN}$ (point $\mathrm{B}$ ), but becomes readily unfavorable for graphene (point A).

The second adsorption energy is then found to decrease as a function of the distance $L$ from the first ion. The value reaches a plateau in energy for large values of $L$, slightly above the first adsorption value. Typically the plateau is reached roughly beyond $\mathrm{L}=3 \AA$ for graphene and beyond $\mathrm{L}=5 \AA$ for BN. Now considering the effect of the solvant, one may observe that it leads to a global destabilizing shift for the second adsorption energy, typically $\sim 0.3-0.5$ $\mathrm{eV}$. The difference in energy between the first adsorption and the plateau is however reduced as compared to vacuum. One may attribute this effect to the screening of charge.

Table 2: Thermodynamic corrections and the Gibbs free energy of the first adsorption evaluated in $\mathrm{eV}$ at $297,15 \mathrm{~K}$ and 1 bar. $\epsilon_{b}$ designates the dielectric permittivity of the solvent, $G\left(O H_{a}\right)$ is the Gibbs free energy of the system with $\mathrm{OH}^{-}$adsorbed on the surface and $G\left(S u b+O H_{f}\right)$ is the Gibbs free energy of the system with a free $\mathrm{OH}^{-}$. All energies are expressed in $\mathrm{eV}$.

\begin{tabular}{lcc}
\hline \hline$O H^{-}$ & BN layer & Graphene layer \\
\hline$T(K)$ & & 297.15 \\
$\epsilon_{b}(P=1$ bar $)$ & & 78.4 \\
\hline \hline$E_{\text {ads } 1}^{\text {sol }}$ & -0.89 & +0.33 \\
Correction & +0.43 & +0.46 \\
$\Delta G_{a d s}(T, P)$ & -0.46 & +0.79 \\
\hline \hline
\end{tabular}

In the following we compute the thermodynamics of adsorption of $\mathrm{OH}^{-}$on the $\mathrm{BN}$ or graphene layers following successful approaches adapted to solid interfaces ${ }^{14}$ (see Supporting Information). The correction terms that link $E_{a d s 1}^{s o l}$ to $\Delta G_{a d s}$ were evaluated at $\mathrm{T}=$ 293.15 K and considering a solute standard concentration $c^{0}=1 \mathrm{~mol} L^{-1}$. The thermody- 
namic corrections are summarized in Table 2 while more details are available in Supporting Information. As a result we find a favorable $\Delta G_{a d s 1}=-0.46 \mathrm{eV}$ for $\mathrm{BN}$ and a non-favorable $\Delta G_{a d s 1}=+0.79 \mathrm{eV}$ for graphene. Thermodynamic corrections also worsen the scenario for the graphene material. This result rules out this charging mechanism on bare graphene but permits to envision it for BN.

We further quantified how the predicted adsorption energies $\Delta G_{a d s 1}$ compare with the surface charge data extracted from the BN measurements in. 1 From the value of $\Delta G_{a d s 1}$, one can define the surface $\mathrm{pK}_{a}$ as

$$
p K_{a}=14+\frac{\Delta G_{a d s 1}}{k_{B} T \times \log (10)} .
$$

As an overall result, Eq. (15) predicts $\mathrm{pK}_{a}=6$ for $\mathrm{BN}$ and $\mathrm{pK}_{a}=27.7$ for graphene. The value for BN agrees well with the post-treatment of experimental data, ${ }^{1}$ in which the electrostatic potential at the surface was evaluated using the Poisson-Boltzmann model. The value proposed for graphene is non-realistic precluding the same mechanism on graphene.

Our results support the strong reactivity of $\mathrm{BN}$ monolayer in the presence of hydroxyl anions contained in saline water. The contrasted result on graphene confirms the differential chemical behavior between the two materials. This echoes the experimental results using nanofluidic tools where much smaller adsorption is found on carbon nanotubes and planar graphitic surface. ${ }^{219}$ Still, the presence of a surface charge on carbon tubes, may suggest that other species than $\mathrm{OH}^{-}$could adsorb onto the graphene surface and charge it.

Another interesting parameter is the maximum coverage for the ions on the surface. Such a quantity enters the global thermodynamics when considering for example the charge-pH relationship in the experiments. The adsorption equilibrium

$$
S u b+O H_{a q}^{-}<=>S u b-O H_{a d s}^{-}
$$


is usually modeled by a Langmuir adsorption model, which introduces a maximal fraction $\theta_{\max }<1$ of $\mathrm{B}$ or $\mathrm{C}$ atoms (for $\mathrm{h}-\mathrm{BN}$ or graphene respectively) that constitute possible adsorption sites. This value for $\theta_{\max }$ fixes the maximal charge expected on the surface, which is an interesting quantity per se. Denoting $\theta$ the fraction of bonded $\mathrm{B}$ or $\mathrm{C}$ atoms to an adsorbed $\mathrm{OH}^{-}$, the chemical potential of $\mathrm{OH}^{-}$at the surface is then modelled as

$$
\mu_{s}=\Delta G_{a d s 1}-e \phi+k_{B} T \log \left(\frac{\theta}{\theta_{\max }-\theta}\right)
$$

where $\phi$ is the electrostatic potential at the surface. The chemical potential of $\mathrm{OH}-$ in the bulk at the concentration $\left[\mathrm{OH}^{-}\right]$expressed in mol. $\mathrm{L}^{-1}$ is fixed at equilibrium by its bulk value.

$$
\mu_{b}=k_{B} T \log \left[\mathrm{OH}^{-}\right]
$$

so that

$$
\frac{\Delta G_{a d s 1}}{k_{B} T}-\frac{e \phi}{k_{B} T}+\log \left(\frac{\theta}{\theta_{\max }-\theta}\right)=\log \left[\mathrm{OH}^{-}\right] .
$$

The equilibrium condition then becomes

$$
\log \left(\frac{\theta}{\theta_{\max }-\theta}\right)=\frac{e \phi}{k_{B} T}+\log (10)\left(p H-p K_{a}\right),
$$

which relates the surface coverage to $\mathrm{pH}$ of the solution and the electrostatic potential at the surface.

A rough estimate for $\theta_{\max }$ can be done for BN on the basis of our DFT calculations. To this aim, we performed preliminary investigations of further adsorption of hydroxyl anions keeping the same minimum favorable distance revealed in Fig. 4. First results are presented on Fig. 5. Considering a third adsorption on the same hexagon leads to an energetically unfavorable configuration (plot B). The adsorption of three adsorbates in a raw is energetically favorable, although the zig zag configuration (plot C) is less favorable than the straight segment (plot D). Finally completing the lines with a fourth adsorbate becomes non-favorable 

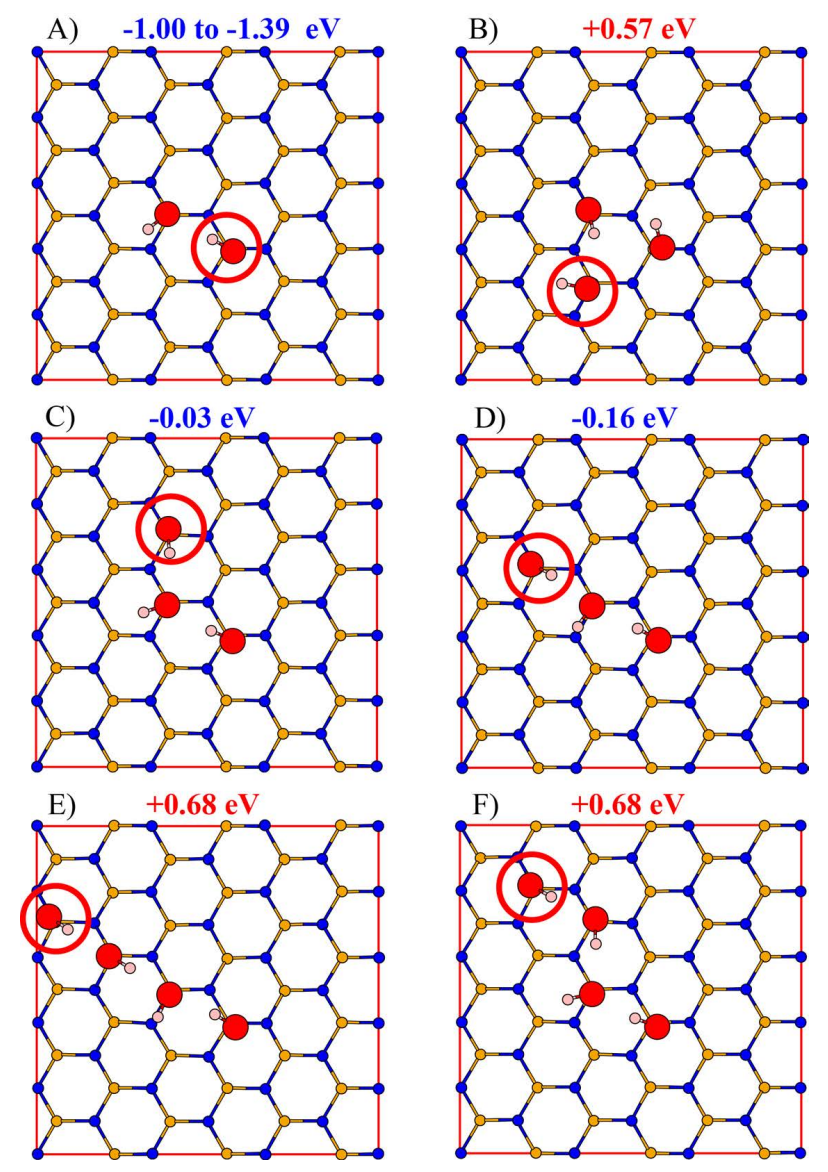

Figure 5: Beyond the second adsorption on BN monolayer : from two adsorbates in the same honeycomb at the minimum favorable distance (A) exploration of three adsorbates (B-C-D) and four adsorbates (E-D) keeping the same favorable distance. The indicated energy is the adsorption energy of the last adsorbate. 
(plots $\mathrm{E}$ and F). Of course these are first configurations and the stability of many other configurations should be evaluated. This goes beyond the present work and will be the object of a forthcoming study. However, on the basis of these results, one may estimate that 3 hydroxides can be grafted at most in a rectangle of $\left(L+l_{3 O H}\right) \times L$, which amounts to a surface of $50 \AA^{2}$. This corresponds to a site density of 0.06 per $\AA^{2}$, and the associated full coverage site density is $\sim 0.18$ per $\AA^{2}$ (30 B atoms per supercell). This leads to $\theta_{\text {max }} \simeq 0.33$ and $\Sigma_{\text {max }} \simeq 0.96 \mathrm{Cm}^{-2}$. These values are in good agreement with the values reported for the maximum charge mesured on BN surfaces. 1 [1

In conclusion, we have investigated comparatively within ab initio DFT framework a monolayer of BN and graphene embedded in implicit water and in contact with one hydroxyl anion. We predict for the first time a contrasted chemical reactivity for graphene and BN : a chemisorption of $\mathrm{OH}^{-}$on $\mathrm{BN}$ while this mechanism is not highlighted on graphene. This striking difference between the two pristine materials is linked to their electronic structure and the lack of screening for graphene surface as compared to the insulating BN. Furthermore, on the basis of atomistic thermodynamics including all the vibrational contributions to energy and entropy, we have derived a free energy of adsorption of - $0.46 \mathrm{eV}$ on BN which corresponds to a $\mathrm{pK}_{a} \simeq 6$, in quantitative agreement with experimental measurements. ${ }^{1}$ Hence this charging scenario is viable on BN and our study leans strong support to the recently-revealed non-inertness of the BN material in contact with aqueous saline phase. On the other hand, the case of graphene requires further investigations. Indeed planar graphitic surfaces show minute surface charge (as deduced from surface conductance measurements) ${ }^{9}$ while the experiments on carbon nanotubes still exhibit a surface charge, ${ }^{2}$ although much weaker as compared to BN tubes. ${ }^{11}$ This suggests the possibility of adsorption of other charged species on the carbon surfaces, not considered in the present study. Future work will involve the exploration at finite $\mathrm{T}$ of the charging mechanism using explicit water solvent and the inclusion of counterions using Ab Initio Molecular Dynamics AIMD, in order to evaluate a 
more realistic $\mathrm{pK}_{a}$ value. Also taking the full stacking of these $2 \mathrm{D}$ materials into account, their curvature effect (since the measurements are made on multi-wall nanotubes of different lengths and diameters) are other ingredients which will be of high interest in understanding the molecular mechanisms at stake in the chemical reactivity of these $2 \mathrm{D}$ materials.

\section{Methods}

\section{Ab initio calculations}

In the VASP code, $17+19$ the wave functions of the system contained in the supercell have been expanded in a plane wave basis set with an energy cutoff of $800 \mathrm{eV}$. The common cutoff for hybrid interfaces is $400 \mathrm{eV}$ in vacuum and taking twice the value is requested for sake of precision when dealing with the implicit solvent scheme (see below).The electronic cores are described by the projector augmented wave method. ${ }^{20}$ The exchange-correlation energies have been evaluated within the generalized gradient approximation (GGA).We employ GGA functionals as suggested by Perdew, Burke, and Ernzerhof (PBE). ${ }^{21 / 22}$ However we have also verified that the key results (adsorption energies) obtained here are not particularly sensitive to the choice of exchange-correlation functional, in particular using the vdW-inclusive optB86b-vdW functional ${ }^{2324}$ which include vdw interactions. We use the smearing technique using a Gaussian broadening of $0.2 \mathrm{eV}$ to achieve electronic convergence. To check the possible influence of the calculation settings, we have modified sequentially the smearing value to $0.1 \mathrm{eV}$, the $\mathrm{k}$-mesh to $3 \mathrm{x} 3 \mathrm{x} 1$ and the vacuum space to $20 \AA$. The adsorption values varie between 0.1 to $0.2 \mathrm{eV}$ but the striking difference between graphene and $\mathrm{BN}$ adsorption energies holds true.

In a second step the solvation energies in implicit water $\left(\mathrm{E}^{\text {sol }}\right)$ are evaluated within the joint density functional theory framework as implemented into VASP by Mathew and Hennig 25 and successfully used recently. $\frac{26}{29}$ In this technique the dielectric permittivity of the medium is defined as a functional of the electronic density. Indeed dielectric permittivity approaches asymptotically the bulk water value at 1 bar, $\epsilon_{b}=78.4$, in the region where the 
electronic density is lower than $\rho_{\text {cut }}=0.0025 \AA^{3}$ (the default cutoff charge density), while $\epsilon_{b}$ $=1$ in the region when the electronic density is high (inside the slab treated as a solute). The cavitation energies which account for the solvant contributions are calculated with a surface tension parameter of $0.525 \mathrm{meV} / \AA^{2}$. The systems are fully optimized in vacuum with atomic forces lower than $0.02 \mathrm{eV}$ per $\AA$. Then the optimized vacuo geometries are subjected to the implicit solvant interaction and the total energies named $\mathrm{E}^{\text {sol }}$ are corrected after an energy and geometry optimization. For the second adsorption $E_{a d s 2}$ however we use the frozen geometry from vacuum for sake of computational time. As a result the adsorption energy in implicit water transforms into :

$$
E_{a d s 1}^{s o l}=E^{s o l}\left(O H_{a}\right)-E^{s o l}\left(S u b+O H_{f}\right)
$$

To evaluate the vibrational entropic corrections to this adsorption energy, we have run a vibrational analysis of state $\mathrm{A}$ and $\mathrm{B}$ (see figure 2) in order to determine the vibrational modes and frequencies. The classical procedure in VASP is to calculate and diagonalize the Hessian matrix using the finite-difference method.

In addition we derived Mulliken atomic charges from a static Dmol3 calculation 30 32 based of the VASP optimized structures in order to understand the spatial location of the excess charge.

\section{Acknowledgement}

B.G., C.P. and M.-L.B. thank the national computational center IDRIS, CINES and TGCC (Grant 2015- [x2015087364]) for the computer grant. L.B. and A.S. thank ANR Blue Energy for support. A.S. and B.G. acknowledge funding from the European Union's H2020 Framework Programme / ERC Starting Grant agreement number 637748 - NanoSOFT. 


\section{Supporting Information Available}

Additional figures and information.

This material is available free of charge via the Internet at http://pubs.acs.org/.

\section{References}

(1) Siria, A.; Poncharal, P.; Biance, A.-L.; Fulcrand, R.; Blase, X.; Purcell, S. T.; Bocquet, L. Giant Osmotic Energy Conversion Measured in a Single Transmembrane Boron Nitride Nanotube. Nature 2013, 494, 455-458.

(2) Secchi, E.; Niguès, A.; Jubin, L.; Siria, A.; Bocquet, L. Scaling Behavior for Ionic Transport and its Fluctuations in Individual Carbon Nanotubes. Phys. Rev. Lett. 2016, $116,154501$.

(3) Novoselov, K. S.; Geim, A. K.; Morozov, S. V.; Jiang, D.; Zhang, Y.; Dubonos, S. V.; Grigorieva, I. V.; Firsov, A. A. Electric Field Effect in Atomically Thin Carbon Films. Science 2004, 306, 666-669.

(4) Sun, Q.; Li, Z.; Searles, D. J.; Chen, Y.; Lu, G. M.; Du, A. Charge-Controlled Switchable CO2 Capture on Boron Nitride Nanomaterials. J. Am. Chem. Soc. 2013, 135, 8246-8253.

(5) Shin, H.; Guan, J.; Zgierski, M. Z.; Kim, K. S.; Kingston, C. T.; Simard, B. Covalent Functionalization of Boron Nitride Nanotubes via Reduction Chemistry. ACS Nano 2015, 9, 12573-12582.

(6) Zhi, C.; Bando, Y.; Terao, T.; Tang, C.; Kuwahara, H.; Golberg, D. Chemically Activated Boron Nitride Nanotubes. Chem Asian J 2009, 4, 1536-1540.

(7) Sainsbury, T.; Satti, A.; May, P.; Wang, Z.; McGovern, I.; Gunko, Y. K.; Coleman, J. 
Oxygen Radical Functionalization of Boron Nitride Nanosheets. J. Am. Chem. Soc. 2012, 134, 18758-18771.

(8) Tang, C.; Bando, Y.; Huang, Y.; Yue, S.; Gu, C.; Xu, F.; ; Golberg, D. Fluorination and Electrical Conductivity of BN Nanotubes. J. Am. Chem. Soc. 2005, 127, 6552-6553.

(9) Radha, B.; Esfandiar, A.; Wang, F. C.; Rooney, A. P.; Gopinadhan, K.; Keerthi, A.; Mishchenko, A.; Janardanan, A.; Blake, P.; Fumagalli, L. et al. Molecular Transport Through Capillaries Made With Atomic-Scale Precision. Nature 2016, 538, 222-225.

(10) Al-Hamdani, Y. S.; Alfè, D.; von Lilienfeld, O. A.; Michaelides, A. Tuning Dissociation Using Isoelectronically Doped Graphene and Hexagonal Boron Nitride: Water and Other Small Molecules. J. Chem. Phys. 2016, 144, 154706.

(11) Graziano, G.; Klimeš, J.; Fernandez-Alonso, F.; Michaelides, A. Improved Description of Soft Layered Materials with Van der Waals Density Functional Theory. J. Phys.: Condens. Matter 2012, 24, 424216.

(12) Ghaderi, N.; Peressi, M. First-Principle Study of Hydroxyl Functional Groups on Pristine, Defected Graphene, and Graphene Epoxide. J. Phys. Chem. C 2010, 114, 2162521630.

(13) Niyogi, S.; Hamon, M. A.; Hu, H.; Zhao, B.; Bhowmik, P.; Sen, R.; Itkis, M. E.; Haddon, R. C. Chemistry of Single-Walled Carbon Nanotubes. Acc. Chem. Res. 2002, 35, 1105-1113.

(14) Reuter, K.; Scheffler, M. Composition and Structure of the $\mathrm{RuO}_{2}(110)$ Surface in an $\mathrm{O}_{2}$ and $\mathrm{CO}$ Environment: Implications for the Catalytic Formation of $\mathrm{CO}_{2}$. Phys. Rev. B 2003, 68, 045407.

(15) Loffreda, D. Theoretical Insight of Adsorption Thermodynamics of Multifunctional Molecules on Metal Surfaces. Surf. Sci. 2006, 600, $2103-2112$. 
(16) Hill, T. An Introduction to Statistical Thermodynamics; Addison-Wesley series in chemistry; Dover Publications, 1960.

(17) Kresse, G.; Hafner, J. Ab Initio Molecular Dynamics for Liquid Metals. Phys. Rev. B 1993, 47, 558-561.

(18) Kresse, G.; Furthmüller, J. Efficiency of Ab-Initio Total Energy Calculations for Metals and Semiconductors Using a Plane-Wave Basis Set. Comput. Mater. Sci. 1996, 6, 1550.

(19) Kresse, G.; Furthmüller, J. Efficient Iterative Schemes for Ab Initio Total-Energy Calculations Using a Plane-Wave Basis Set. Phys. Rev. B 1996, 54, 11169-11186.

(20) Kresse, G. From Ultrasoft Pseudopotentials to the Projector Augmented-Wave Method. Phys. Rev. B 1999, 59, 1758-1775.

(21) Perdew, J. P.; Burke, K.; Ernzerhof, M. Generalized Gradient Approximation Made Simple. Phys. Rev. Lett. 1996, 77, 3865-3868.

(22) Ziesche, P.; Kurth, S.; Perdew, J. P. Density Functionals from LDA to GGA. Comput. Mater. Sci. 1998, 11, 122-127.

(23) Klimeš, J.; Bowler, D. R.; Michaelides, A. Van der Waals Density Functionals Applied to Solids. Phys. Rev. B 2011, 83, 195131.

(24) Klimeš, J.; Michaelides, A. Perspective: Advances and Challenges in Treating Van der Waals Dispersion Forces in Density Functional Theory. J. Chem. Phys. 2012, 137, 120901.

(25) Mathew, K.; Sundararaman, R.; Letchworth-Weaver, K.; Arias, T. A.; Hennig, R. G. Implicit Solvation Model for Density-Functional Study of Nanocrystal Surfaces and Reaction Pathways. J. Chem. Phys. 2014, 140, 084106. 
(26) Lespes, N.; Filhol, J.-S. Using Implicit Solvent in Ab Initio Electrochemical Modeling: Investigating $\mathrm{Li}^{+} / \mathrm{Li}$ Electrochemistry at a $\mathrm{Li} /$ Solvent Interface. J. Chem. Theory Comput. 2015, 11, 3375-3382.

(27) Sakong, S.; Naderian, M.; Mathew, K.; Hennig, R. G.; Gro A. Density Functional Theory Study of the Electrochemical Interface Between a Pt Electrode and an Aqueous Electrolyte using an Implicit Solvent Method. J. Chem. Phys. 2015, 142, 234107.

(28) Steinmann, S. N.; Michel, C.; Schwiedernoch, R.; Filhol, J.-S.; Sautet, P. Modeling the $\mathrm{HCOOH} / \mathrm{CO}_{2}$ Electrocatalytic Reaction: When Details Are Key. ChemPhysChem 2015, 16, 2307-2311.

(29) Garcia-Ratés, M.; López, N. Multigrid-Based Methodology for Implicit Solvation Models in Periodic DFT. J. Chem. Theory Comput. 2016, 12, 1331-1341.

(30) Delley, B. An All-Electron Numerical Method for Solving the Local Density Functional for Polyatomic Molecules. J. Chem. Phys. 1990, 92, 508.

(31) Delley, B. From Molecules to Solids with the DMol ${ }^{3}$ Approach. J. Chem. Phys. 2000, $113,7756$.

(32) Delley, B. Fast Calculation of Electrostatics in Crystals and Large Molecules. J. Phys. Chem. 1996, 100, 6107-6110. 